\title{
Minimum Energy Problems in Hilbert Function Space
}

\author{
by MARSHALL C. Y. KUO
}

Department of Electrical Engineering

Newark College of Engineering, Newark, New Jersey

and LOUIS F. KAZDA

Department of Electrical Engineering

University of Michigan, Ann Arbor, Michigan

ARSTRACT: Minimum energy problems in Hilbert function space are formulated. The methods presented are applicable to both continuous and discrete linear systems. Transformation of a particular operator equation into a set of $2 n$ linear differential equations is described. Applications to physical systems are illustrated.

\section{Introduction}

The term "minimum energy" is derived from the fact that many physical problems involving energy can be formulated such that the signals $u(t)$ involved are considered as elements in a Hilbert function space of functions of $t$ with the energy of the signal being

$$
\|u\|^{2}=\langle u, u\rangle=\int|u(t)|^{2} d t
$$

In a fairly obvious manner this concept may be generalized to the case such that the energy of signals may be described by

$$
\|u\|_{H_{1}}^{2}+\|F u\|_{H_{2}}^{2}
$$

where the input signal $u \in H_{1}$ and the output signal $x \in H_{2}$ are related by $x=F u \in H_{2}$.

The methods presented here are strongly motivated by and closely related to the previous work of Balakrishnan (1) and Porter (2). It is the purpose of this paper to generalize the concept of minimum energy problems from the functional analysis view of point with emphasis on both mathematical theories and physical applications.

\section{Statement of Problem}

Let $H_{1}$ and $H_{2}$ be two Hilbert function spaces and $F$ be a transfer operator carrying $H_{1}$ into $H_{2}$. Let $F^{\iota}$ be a bounded linear operator of $H_{1}$ into $R^{n}$ and is 
defined by

$$
\left.F^{t}=\sum_{i=1}^{n} \phi_{i}(t)\right\rangle\left\langle f_{i}^{t}\right.
$$

where the set of range vectors, $\left\{\phi_{i}\right\}_{1}^{n}$, and the set of functionals, $\left\{\left\langle f_{i}\right\}_{1}^{n}\right.$, are assumed to be linearly independent. If $x(t)$ is an arbitrary element of $R^{n}$ and $\hat{u}$ and $\hat{x}$ are arbitrary elements of $H_{1}$ and $H_{2}$, respectively, from the set $U_{x} \subset H_{1}$ defined by

$$
U_{x}=\left\{u: u \in H_{1}, F^{t} u=x(t)\right\}
$$

find the element $u$ which minimizes the functional $J(u)$ where

$$
\begin{aligned}
& J(u)=\|u\|_{H_{1}}^{2} \\
& J(u)=\|F u\|_{H_{2}}^{2}+\|u\|_{H_{1}}^{2} \\
& J(u)=\|F u-\hat{x}\|_{H^{2}}^{2}+\|u-\hat{u}\|_{H_{1}}^{2} .
\end{aligned}
$$

\section{Remarks:}

1) The phrase "Hilbert function space" is defined over a finite interval $\tau=\left[t_{0}, T\right]$ of reals, or $\sigma=\left[t_{0}, t_{1} \cdots t_{f}\right]$ to be $L_{2}(\tau)$, or $l_{2}(\sigma)$, respectively.

2) $F^{t} u$ is the value of $F u$ at a point when the time is equal to $t$ and $F u$ is the unique continuous image of $u$ under $F$.

\section{Definitions and Theorems}

In order to deal more concisely with the concept of generalized minimum energy problems the results of the solutions are summarized in the following theorems:

\section{Theorem I.}

Let $M$ denote the linear manifold spanned by the set $\left\{f_{i}{ }^{t}\right\}_{1}^{n}$, then $M+$, the orthogonal complement of $M$ in $H_{1}$, is the null space of $F^{t}$ and the restriction of the opcrator $F^{t}$ to $M$ is nonsingular.

\section{Proof:}

Let $N_{F}{ }^{t}$ denote the null space of $F^{t}$. If $u \in M \perp$ then $\left\langle f_{i}{ }^{t}, u\right\rangle=0, i=1 \cdots n$ and hence $F^{t} u=0$ which implies that $u \in N_{F}^{t}$ hence $M^{\perp} \subset N_{F}^{t}$. On the other hand if $u \in N_{F}$ then

$$
\left.F^{t} u=\sum_{i=1}^{n} \phi_{i}\right\rangle\left\langle f_{i}^{t}, u\right\rangle=0
$$

and since the set $\left\{\phi_{i}\right\}_{1}^{n}$ is linearly independent $\left\langle f_{i} t, u\right\rangle=0, i=1, \cdots, n$, which implies that $u \in M \perp$ hence $M \perp \supset N_{F}{ }^{t}$ and the first part of Theorem I is proved. Let $F_{M}$ denote the restriction of $F^{t}$ to the subspace $M$ that $F_{M}$ is nonsingular 
(3) is immediate from the fact that $F_{M}$ is a linear mapping between finitedimensional spaces with a domain and range of equal dimensions.

\section{Theorem II.}

The set $U_{x}$ has a unique element in $M$ which is exactly $u^{0}$, the solution of the problem for the minimization of the functional given by Eq. 5 .

\section{Proof:}

Since $F_{M}$ is nonsingular $F_{M}^{-1}$ exists and the element defined by $u=F_{M}^{-1} x$ is the unique preimage of $x$ in $M$. Since $M$ is finite dimensional it is closed and we may consider the orthogonal decomposition $H_{1}=M+M+$ of $H_{1}$. Let $u^{0}$ denote an arbitrary element of $H_{1}$ which may then be uniquely expressed as

$$
u^{0}=u_{1}+u_{2}
$$

where $u_{1} \in M$ and $u_{2} \in M \perp$. Since $M \perp$ is the null space of $F^{t}$ the additional relation

$$
x(t)=F^{t} u^{0}=F^{t}\left(u_{1}+u_{2}\right)=F^{t} u_{1}+F^{t} u_{2}=F^{t} u_{1}+0
$$

holds. Since $u_{1}$ is a preimage of $x(t)$ in $M$ it is unique and given by

From the relation

$$
u_{1}=F_{M}^{-1} x
$$

it is apparent that

$$
\left\|u^{0}\right\|^{2}=\left\|F_{M}^{-1} x\right\|^{2}+\left\|u_{2}\right\|^{2}
$$

$$
\left\|u^{0}\right\|^{2} \geq\left\|F_{M}^{-1} x\right\|^{2}
$$

holds for every other $u^{0} \in H_{1}$ with equality if and only if $u^{0}=F_{M}{ }^{-1} x$ and hence the theorem is proved.

I et $x(t) \in R^{n}$ be a given arbitrary vector and take the set $\left\{f_{i}^{t}\right\}_{1}^{n}$ as a basis for $M$. Then $u^{0}$ may be expressed by

$$
u^{0}=\sum_{i=1}^{\infty} c_{i} f_{i}
$$

where $c_{i}$ is a scalar quantity to be determined. It can be shown (2) that

$$
c=F^{-1} \beta,
$$

where $\mathfrak{F}^{-1}$ is the inverse matrix of $\mathcal{F}$ which is defined by

$$
\begin{aligned}
\mathcal{F} & =\left[\left\langle f_{i}{ }^{t}, f_{j}{ }^{t}\right\rangle\right], \\
\beta & =\text { col. }\left(\beta_{1} \cdots \beta_{n}\right), \quad \beta_{i}=\left\langle\phi_{i}{ }^{+}, x\right\rangle, \\
c & =\text { col. }\left(c_{1} \cdots c_{n}\right) .
\end{aligned}
$$


Thus

$$
J^{0}=\left\|u^{0}\right\|_{H_{1}}^{2}=\left\langle\beta, \mathcal{F}^{-1} \beta\right\rangle
$$

\section{Theorem III.}

The element $u^{0}$ which minimizes the functional Eq. 6 is given by

$$
u^{0}=\left(I+F^{*} F\right)^{-1} v, \quad v \in M \text {. }
$$

From the operation of adjoint operator Eq. 6 may be rewritten as follows:

$$
J(u)=\left\langle\left(I+F^{*} F\right) u, u\right\rangle_{H_{1}}
$$

Since $I+F^{*} F$ is a positive operator it can be expressed as the product of an operator $K$ and its adjoint operator $K^{*}$, i.e.,

$$
I+F^{*} F=K^{*} K, \quad \text { where } K^{-1} \text { exists. }
$$

From Eqs. 17 and 18, we obtain

$$
J(u)=\langle K u, K u\rangle_{H_{2}}
$$

Let $w=K u \in H_{2}$ and let $G^{t}$ denote the linear transformation of $H_{2}$ onto $R^{n}$ with the property that

$$
G^{t} w=F^{t} u=x(t)
$$

and

$$
\left.G^{t}=\sum_{i=1}^{n} \phi_{i}\right\rangle\left\langle g_{i}{ }^{t}\right.
$$

It is clear that $G^{t}$ is linear and well defined on $H_{2}$. Thus, if $x(t) \in R^{n}$ and if $w \in H_{2}$ is the element with minimum norm mapping into $x(t)$ under $G^{t}$ then $u=K^{-1} w$ is the $u \in H_{1}$ which minimizes

$$
J(u)=\|F u\|_{H_{2}}^{2}+\|u\|_{H_{1}}^{2} .
$$

While doing so the problem of minimization of the functional Eq. 6 reduces to the problem of minimization of Eq. 5 with

$$
G^{t}: H_{2} \rightarrow R^{n}
$$

being the operator in question.

\section{Proof of Theorem III:}

Let $Q$ denote the linear manifold spanned by the set $\left\{g_{i}\right\}_{1}^{n}$. By applying 
Theorems I and II the element $w^{0}$ with minimum norm is given by

$$
w^{0}=\sum_{i=1}^{n} c_{i} g_{i}{ }^{t}
$$

where $c_{i}$ is a constant to be determined.

From Eqs. 3, 20 and 21, we obtain

$$
\left.\left.\left.G^{t} w=\sum_{i=1}^{n} \phi_{i}\right\rangle\left\langle g_{i}^{t}, w\right\rangle=\sum_{i=1}^{n} \phi_{i}\right\rangle\left\langle K^{*} g_{i}{ }^{t}, u\right\rangle=\sum_{i=1}^{n} \phi_{i}\right\rangle\left\langle f_{i} t, u\right\rangle
$$

Since the set $\left\{\phi_{i}\right\}_{1}^{n}$ is linearly independent and $u=0$ is a trivial solution it follows from Eq. 23 that

$$
f_{i}^{t}=K^{*} g_{i}{ }^{t}
$$

From Eqs. 22 and 24, there results

$$
K^{*} w^{0}=\sum_{i=1}^{n} c_{i} f_{i}
$$

Thus

or

$$
K^{*} K u^{0}=v, \quad v \in M
$$

$$
u^{0}=\left(I+F^{*} F^{-1} v, \quad v \in M .\right.
$$

\section{Lemma 1.}

If $F$ is a linear transformation of $H_{1}$ into $H_{2}$ then $I+F^{*} F$ is nonsingular.

\section{Proof:} itself.

We must show that $I+F^{*} F$ is one to one onto as a mapping of $H_{1}$ into

(i) It is one to one, because $\left(I+F^{*} F\right) u=0, u \in H_{1}$, implies that (4)

$$
\begin{aligned}
\mathbf{0} & =\left\langle\left(I+F^{*} F\right) u, u\right\rangle_{H_{1}} \\
& =\langle u, u\rangle_{H_{1}}+\left\langle F^{*} F u, u\right\rangle_{H_{1}} \\
& =\langle u, u\rangle_{H_{1}}+\langle F u, F u\rangle_{H_{2}} \\
& =\|u\|_{H_{1}}^{2}+\|F u\|_{H_{2}}^{2}(5)
\end{aligned}
$$

from which we have $u=0$.

(ii) We next show that the range $R$ of $I+F^{*} F$ is close. It follows from

that

$$
\left\|\left(I+F^{*} F\right) u\right\|_{H_{1}}^{2}=\|u\|_{H_{1}}^{2}+\left\|F^{*} F u\right\|_{H_{1}}^{2}+2\left\langle F^{*} F u, u\right\rangle_{H_{1}}
$$

$$
\left\|\left(I+F^{*} F\right) u\right\|_{H_{1}} \geq\|u\|_{H_{1}},
$$

since $F^{*} F$ is positive. By this inequality and the completeness of $H_{1}, R$ is com- 
plete and therefore closed. We conclude the proof that $R=H_{1}$; for otherwise there would exist a nonzero vector $u_{0}$ orthogonal to $R$ and this contradicts the fact that

$$
\left\langle\left(I+F^{*} F\right) u_{0}, u_{0}\right\rangle_{H_{1}}=0 \Rightarrow\left\|u_{0}\right\|_{H 1}^{2}=-\left\langle F^{*} F u_{0}, u_{0}\right\rangle_{H_{1}}<0 \Rightarrow u_{0}=0
$$

\section{Definition:}

Let $F$ be a linear mapping of $H_{1}$ into $H_{2}$. The set $H(F)$ defined by $H(F)=$ $\left\{(u, v): v=F u, u \in H_{1}\right\}$ is called the graph of $F$. The graph of a transformation is thus the subset of

$$
H_{1} \times H_{2}=\left\{(u, v): u \in H_{1}, v \in H_{2}\right\}
$$

for which the ordinate $v$ is related the abscissa $u$ by $v=F u$. The basic properties pertinent to the present undertaking are summarized by the following lemmas:

\section{Lemma 2.}

If addition and scalar multiplication are defined on the set $H(F)$ by

$$
\begin{aligned}
k(f, g) & =(k f, k g) \\
\left(f_{1}, g_{1}\right)+\left(f_{2}, g_{2}\right) & =\left(f_{1}+f_{2}, g_{1}+g_{2}\right)
\end{aligned}
$$

then $H(F)$ is a linear space.

\section{Lemma 3.}

If $H_{1}$ and $H_{2}$ are Hilbert spaces and an inner product on $H(F)$ is defined by

$$
\left\langle\left(f_{1}, g_{1}\right),\left(f_{2}, g_{2}\right)\right\rangle_{H(F)}=\left\langle f_{1}, f_{2}\right\rangle_{H_{1}}+\left\langle g_{1}, g_{2}\right\rangle_{H_{2}}
$$

then $H(F)$ is a Hilbert space.

\section{Lemma 4.}

If $F$ is bounded then $H(F)$ is a closed linear subspace of $H_{1} \times H_{2}$.

The above lemmas will be proved in Appendix A.

\section{Theorem IV.}

If $(\mathfrak{a}, \hat{x}) \in H(F)$ then the element $u^{0}$ which minimizes the functional Eq. 7 is satisfied by

$$
\left(I+F^{*} F\right)\left(u^{0}-\hat{u}\right)=v, \quad v \in M .
$$

\section{Proof:}

Since $(\mathfrak{u}, \hat{x}) \in H(F)$

$$
\hat{x}=F \hat{u} .
$$


Let $u-\hat{u}=\bar{u}$. Then the functional Eq. 7 may be written as

$$
J(\bar{u})=\|F \bar{u}\|_{H 2}^{2}+\|\bar{u}\|_{H 1}^{2}
$$

It follows directly from Theorem III that

$$
\left(I+F^{*} F\right) \bar{u}=v, \quad v \in M
$$

\section{Theorem $V$.}

If $(\hat{u}, \hat{x}) \in H_{1} \times H_{2}$ then the element $u^{0}$ which minimizes the functional Eq. 7 is given by

$$
u^{0}=\left(I+F^{*} F\right)^{-1}\left(v+\hat{u}+F^{*} \hat{x}\right), \quad v \in M
$$

Since $H(F)$ is a closed linear subspace of $H_{1} \times H_{2}$ it follows that the decomposition

$$
H_{1} \times H_{2}=H(F)+H(F) \perp
$$

may be defined along with the orthogonal projection $P_{I}$ of $H_{1} \times H_{2}$ on $H\left(F^{\prime}\right)$. Let $(\hat{u}, \hat{x})$ be defined by

$$
(\bar{u}, \bar{x})=P_{H}(\hat{u}, \hat{x})
$$

then $(\bar{u}, \bar{x})$ is in $H(F)$ and $(\hat{u}, \hat{x})-(\bar{u}, \bar{x}) \in H(F) \perp$ and the

$$
\begin{aligned}
\|F u-\hat{x}\|_{H 2}^{2}+\|u-\hat{u}\|_{H 1}^{2} \\
\quad=\|(F u-\bar{x})+(\bar{x}-\hat{x})\|_{H 2}^{2}+\|(u-\bar{u})+(\bar{u}-\hat{u})\|_{H 1}^{2} \\
\quad=\|F u-\bar{x}\|_{H 2}^{2}+\|\bar{x}-\hat{x}\|_{H 2}^{2}+\|u-\bar{u}\|_{H 1}^{2}+\|\bar{u}-\hat{u}\|_{H 1}^{2} \\
\quad=\|F u-\bar{x}\|_{H_{2}}^{2}+\|u-\bar{u}\|_{H 1}^{2}+\|\bar{x}-\hat{x}\|_{H 2}^{2}+\|\bar{u}-\hat{u}\|_{H 1}^{2}
\end{aligned}
$$

holds for all $u \in H_{1}$. The last two terms are independent of $u$ and hence it is apparent that Theorem $V$ reduces to the case of Theorem IV by taking the projection of $(\hat{u}, \hat{x})$ on the graph of $F$.

Let the operator $V$ of the Hilbert space $\mathrm{H}_{1} \times \mathrm{H}_{2}$ be defined by the relation

$$
V(u, v)=(-v, u), \quad(u, v) \in H_{1} \times H_{2} .
$$

It can be proved (see Appendix B) that the orthogonal complement of $H(F)$ in $H_{1} \times H_{2}$ is given by

$$
H(F) \perp=V H\left(F^{*}\right)
$$

A consequence of this result is that the orthogonal decomposition of $\mathrm{H}_{1} \times \mathrm{H}_{2}$ on $H(F)$ is given explicitly by the formula (6)

$$
H_{1} \times H_{2}=H(F)+V H\left(F^{*}\right) .
$$


This formula will prove useful in defining the orthogonal projection of $\mathrm{H}_{1} \times \mathrm{H}_{2}$ on $H(F)$.

Let $(\hat{u}, \hat{x}) \in H_{1} \times H_{2}$ denote an arbitrary vector. Then in view of Eq. 44 a unique decomposition exists of the form

$$
\begin{aligned}
(\hat{u}, \hat{x}) & =\bar{u}, F \bar{u})+V\left(u_{1}, F^{*} u_{1}\right) \\
& =(\bar{u}, F \bar{u})+\left(-F^{*} u_{1}, u_{1}\right) \\
& =\left(\bar{u}-F^{*} u_{1}, F \bar{u}+u_{1}\right) .
\end{aligned}
$$

This equality implies the two equalities

and

$$
\hat{u}=\bar{u}-F^{*} u_{1}
$$

$$
\hat{x}=F \bar{u}+u_{1}
$$

Operating on the second equation with $F^{*}$ and using the result to eliminate $F^{*} u_{1}$ in the first equation produces the result

$$
\vec{u}=\left(I+F^{*} F\right)^{-1}\left(\hat{u}+F^{*} \hat{x}\right) .
$$

Replacing $\hat{u}$ in Eq. 35 by $\bar{u}$ expressed in Eq. 47 gives

$$
\left(I+F^{*} F\right)\left[u^{0}-\left(I+F^{*} F\right)^{-1}\left(\hat{u}+F^{*} \hat{x}\right)\right]=v, \quad v \in M
$$

or

$$
u^{0}=\left(I+F^{*} F\right)^{-1}\left(v+\hat{u}+F^{*} \hat{x}\right), \quad v \in M .
$$

\section{A Method of Evaluating the Optimal Element $\boldsymbol{u}^{0}$ without Inversion of Operator}

It was shown that the optimal element $u^{0}$ which minimizes the functional Eq. 7 is given by Eq. 49. Therefore, the evaluation of $u^{0}$ involves the inversion of $\left(I+F^{*} F\right)$. However, there is a simple method of finding the element $u^{0}$ which avoids the inversion of the operator $\left(I+F^{*} F\right)$ if the operator $F^{t}$ is defined by (7)

$$
F^{t} u=\int_{t_{0}}^{t} \Phi\left(t, t_{0}\right) B(s) u(s) d s
$$

which is a bounded linear operator and is well known to be the solution of the system equation as given by

$$
\dot{x}(t)=x(t) A(t)+B(t) u(t), \quad x\left(t_{0}\right)=0 .
$$

This method is carried out by transforming the operator Eq. 49 into $2 n$ linear differential equations. 


\section{Marshall C. Y. Kuo and Louis F. Kazda}

Since $v \in M$ has a unique expansion in terms of any basis for that space. In the present instance the rows of matrix $\Phi\left(t_{0}, t\right) B(t)$ provide a convenient basis. By introducing the scalar $c=$ col. $\left(c_{1}, c_{2} \cdots c_{n}\right)$ we may write

$$
v(t)=B^{*}(t) \Phi^{*}\left(t_{0}, t\right) c
$$

as a defining relation for an arbitrary $v \in M$. Along the optimal trajectory $x=F u^{0} \mathrm{Eq} .49$ may be written as

$$
u^{0}=\hat{u}+v+F^{*}(\hat{x}-x)
$$

It is not difficult to show (see Appendix C) that

$$
\left(F^{*} x\right)(t)=B^{*}(t) \Phi^{*}\left(t_{0}, t\right) \int_{t}^{T} \Phi^{*}\left(s, t_{0}\right) x(s) d s
$$

for $t \in \tau=\left[t_{0}, T\right]$ is the defining equation for $F^{*}$ as $t$ sweeps through $\tau$. Letting $\alpha(t)$ denote the tuplet

$$
\begin{aligned}
\alpha(t) & =c-\int_{t}^{T} \Phi^{*}\left(s, t_{0}\right)[x(s)-\hat{x}(s)] d s \\
& =c-\int_{t_{0}}^{T} \Phi^{*}\left(s, l_{0}\right)[x(s)-\hat{x}(s)] d s+\int_{t_{0}}^{t} \Phi^{*}\left(s, t_{0}\right)[x(s)-\hat{x}(s)] d s \\
& =\alpha\left(t_{0}\right)+\int_{t_{0}}^{t} \Phi^{*}\left(s, t_{0}\right)[x(s)-\hat{x}(s)] d s .
\end{aligned}
$$

It is apparent that Eq. 53 may be written in the form

$$
u^{0}-\hat{u}=B^{*}(t) \Phi^{*}\left(t_{0}, t\right) \alpha(t)
$$

Consider now the vector $\lambda(t)=\Phi^{*}\left(t_{0}, t\right) \alpha(t)$. Using the property that $\Phi^{*}\left(t_{0}, t\right)=$ $\Psi\left(t, t_{0}\right)$, where $\Psi\left(t, t_{0}\right)$ is the transition matrix for the adjoint system

$$
\dot{\Psi}\left(t, t_{0}\right)=-A^{*}(t) \Psi\left(t, t_{0}\right), \quad \Psi\left(t_{0}, t_{0}\right)=I
$$

( $I$ is the identity matrix), it is apparent from Eq. 55 that

$$
\begin{aligned}
\lambda(t) & =\Phi^{*}\left(t_{0}, t\right) \alpha\left(t_{0}\right)+\Phi^{*}\left(t_{0}, t\right) \int_{t_{0}}^{t} \Phi^{*}\left(s, t_{0}\right)[x(s)-\hat{x}(s)] d s \\
& =\Psi\left(t, t_{0}\right) \alpha\left(t_{0}\right)+\Psi\left(t, t_{0}\right) \int_{t_{0}}^{t} \Psi\left(t_{0}, s\right)[x(s)-\bar{x}(s)] d s
\end{aligned}
$$


which implies immediately that $\lambda(t)$ is the solution to the differential system

$$
\dot{\lambda}(t)=-A^{*}(t) \lambda(t)+[x(t)-\hat{x}(t)], \quad \lambda\left(t_{0}\right)=\lambda_{0}
$$

The original differential system for this example along the optimal trajectory is given by

$$
\begin{aligned}
\dot{x}(t) & =A(t) x(t)+B(t) u^{0}(t) \\
& =A(t) x(t)+B(t)\left[B^{*}(t) \lambda(t)+\hat{u}\right] .
\end{aligned}
$$

Thus, we have the $2 n$ linear differential equations

$$
\dot{x}(t)=A(t) x(t)+B(t)\left[B^{*}(t) \lambda(t)+\hat{u}\right], \quad x\left(t_{0}\right)=x_{0}
$$

and

$$
\dot{\lambda}(t)=x(t)-\hat{x}(t)-A^{*}(t) \lambda(t), \quad \lambda\left(t_{0}\right)=\lambda_{0},
$$

the solution that determines $\lambda(t)$, which in turn determines $u^{0}(t)$ by

$$
u^{0}(t)=B^{*}(t) \lambda(t)+\hat{u}
$$

One important point to be noted is that $\lambda_{0}$ is unknown. Indeed, $\lambda_{0}$ is a function of $c$ which we originally set out to determine. The solution of these equations may be expressed in terms of the transition matrix $\theta\left(t, t_{0}\right)$ which is defined by

$$
\dot{\theta}\left(t, t_{0}\right)=\left[\begin{array}{c:c}
A(t) & B(t) B^{*}(t) \\
\hdashline I & -A^{*}(t)
\end{array}\right] \Theta\left(t, t_{0}\right)
$$

with

$$
\Theta\left(t_{0}, t_{0}\right)=I
$$

The transition matrix has $2 n$ rows and $2 n$ columns. It can be partitioned into four $n \times n$ submatrices

$$
\theta\left(t, t_{0}\right)=\left[\begin{array}{c:c}
\theta_{11}\left(t, t_{0}\right) & \theta_{12}\left(t, t_{0}\right) \\
\hdashline \theta_{21}\left(t, t_{0}\right) & \theta_{22}\left(t, t_{0}\right)
\end{array}\right]
$$

Solution to the system Eq. 61 thus can be expressed by

where

$$
\left[\begin{array}{l}
x(t) \\
\lambda(t)
\end{array}\right]=\left[\begin{array}{ll}
\Theta_{11}\left(t, t_{0}\right) & \Theta_{12}\left(t, t_{0}\right) \\
\Theta_{21}\left(t, t_{0}\right) & \Theta_{22}\left(t, t_{0}\right)
\end{array}\right]\left[\begin{array}{l}
x\left(t_{0}\right) \\
\lambda\left(t_{0}\right)
\end{array}\right]+\left[\begin{array}{l}
q_{1}\left(t, t_{0}\right) \\
q_{2}\left(t, t_{0}\right)
\end{array}\right]
$$

$$
\left[\begin{array}{l}
q_{1}\left(t, t_{0}\right) \\
q_{2}\left(t, t_{0}\right)
\end{array}\right]=\int_{t_{0}}^{t}\left[\begin{array}{ll}
\theta_{11}(t, s) & \theta_{12}(t, s) \\
\theta_{21}(t, s) & \theta_{22}(t, s)
\end{array}\right]\left[\begin{array}{c}
B(s) \hat{u}(s) \\
-\hat{x}(s)
\end{array}\right] d s .
$$


In particular we have

$$
x(T)=\theta_{11}\left(T, t_{0}\right) x\left(t_{0}\right)+\theta_{12}\left(T, t_{0}\right) \lambda\left(t_{0}\right)+q_{1}\left(T, t_{0}\right) .
$$

Thus, if $x(T)$ and $x\left(t_{0}\right)$ are known this expression determines $\lambda\left(t_{0}\right)$. If $x\left(t_{0}\right)=0$ and $\theta_{12}\left(t, t_{0}\right)$ is nonsingular then

$$
\lambda\left(t_{0}\right)=\theta_{12}^{-1}\left(T, t_{0}\right)\left[x(T)-q_{1}\left(T, t_{0}\right)\right] .
$$

This gives

$$
\lambda(t)=\Theta_{22}\left(t, t_{0}\right) \Theta_{12}\left(T, t_{0}\right)^{-1}\left[x(T)-q_{1}\left(T, t_{0}\right)\right]+q_{2}\left(t, t_{0}\right)
$$

Substituting Eq. 68 into Eq. 56 gives

$$
u^{0}(t)=B^{*}(t)\left\{\theta_{22}\left(t, t_{0}\right) \theta_{12}\left(T, t_{0}\right)^{-1}\left[x(T)-q_{1}\left(T, t_{0}\right)\right]+q_{2}\left(t, t_{0}\right)\right\}+\hat{u} .
$$

\section{Physical Applications}

\section{Example I.}

Consider the continuous second-order position control servomechanism shown in Fig. 1 where the gain, mass, and viscous damping which, in general, are

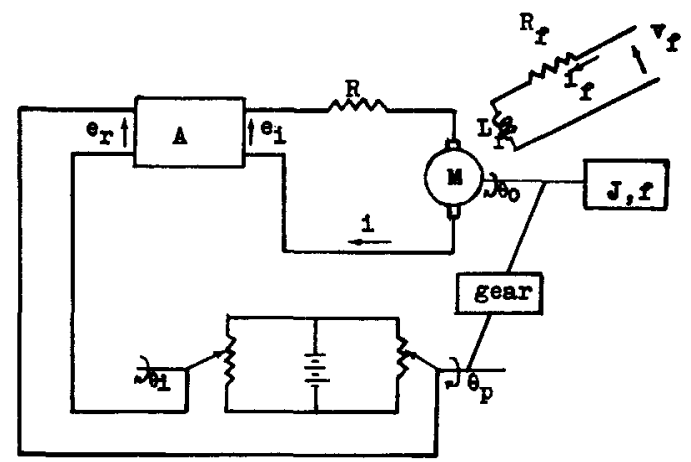

Fig. 1. Second-order position control servomechanism.

deterministic differentiable functions of time are assumed to be constant. The motor armature inductance and backlash of the gear are neglected. Now, by fixing $i_{f}$ the following basic equations describe this system:

$$
\begin{aligned}
e_{i} & =A e_{r}=A k_{4}\left(\theta_{i}-\theta_{p}\right)=R i+k_{1} \dot{\theta}_{0} \\
T & =k_{2} i=J \ddot{\theta}_{0}+f \dot{\theta}_{0} \\
\theta_{p} & =k_{3} \theta_{0}
\end{aligned}
$$


where $A=$ gain of amplifier

$k_{1}=$ motor constant

$T, k_{2}=$ developed torque, torque constant

$k_{3}=$ gear ratio

$k_{4}=$ proportionality constant of potentiometers

$\phi=$ air gap flux of motor

$R=$ resistance of armature circuit of servomotor $M$

$R_{f}, L_{f}=$ resistance, inductance of motor field circuit

$J, f=$ inertia, viscous damping referred to motor shaft

It is desired to find $\theta_{i}(t)$ such that

$$
\theta_{0}\left(t_{0}\right)=\theta_{0}, \quad \dot{\theta}_{0}\left(t_{0}\right)=\dot{\theta}_{0}, \quad \theta_{0}(T)=\theta_{T}, \quad \dot{\theta}_{0}(T)=\dot{\theta}_{T}
$$

and

$$
J=\int_{t_{0}}^{T} \theta_{i}{ }^{2} d t \text { has a minimum value. }
$$

From Eqs. 70 and 71 we have

$$
\frac{J R}{k_{2}} \ddot{\theta}_{0}+\left(\frac{f R}{k_{2}}+k_{1}\right) \dot{\theta}_{0}+A k_{4} k_{3} \theta_{0}=A k_{4} \theta_{i}
$$

or

$$
\ddot{\theta}_{0}+a_{1} \dot{\theta}_{0}+a_{0} \theta_{0}=d \theta_{i}
$$

where

$$
\begin{aligned}
& a_{1}=\frac{k_{2}}{J R}\left(\frac{f R}{k_{2}}+k_{1}\right) \\
& a_{0}=\frac{A k_{2} k_{3} k_{4}}{J R} \\
& d=A k_{2} k_{4}\left(\frac{1}{J R}\right) .
\end{aligned}
$$

Let $x_{1}(t)=\theta_{0}(t), x_{2}(t)=\dot{\theta}_{0}(t)$ and $u(t)=\theta_{i}(t)$. Then in matrix notation Eq. 75 may be written as

$$
\dot{x}(t)=A x(t)+B u(t)
$$

where

$$
A=\left[\begin{array}{cc}
0 & 1 \\
-a_{0} & -a_{1}
\end{array}\right] \text { and } B=\left[\begin{array}{l}
0 \\
d
\end{array}\right] \text {. }
$$


The transition matrix $\Phi\left(t, t_{0}\right)$ of the system, Eq. 76, is given by

where

$$
\Phi\left(t, t_{0}\right)=\left[\begin{array}{ll}
\phi_{11}\left(t, t_{0}\right) & \phi_{12}\left(t, t_{0}\right) \\
\phi_{21}\left(t, t_{0}\right) & \phi_{22}\left(t, t_{0}\right)
\end{array}\right]
$$

$$
\begin{gathered}
\phi_{11}\left(t, t_{0}\right)=c_{1} \exp \left[-b\left(t-t_{0}\right)\right] \sin \left[w\left(t-t_{0}\right)+\psi_{1}\right] \\
\phi_{12}\left(t, t_{0}\right)=c_{2} \exp \left[-b\left(t-t_{0}\right)\right] \sin \left[w\left(t-t_{0}\right)\right] \\
\phi_{21}(t, t)=c_{3} \exp \left[-b\left(t-t_{0}\right)\right] \sin \left[w\left(t-t_{0}\right)\right] \\
\phi_{22}\left(t, t_{0}\right)=c_{4} \exp \left[-b\left(t-t_{0}\right)\right] \sin \left[w\left(t-t_{0}\right)+\psi_{2}\right] \\
c_{1}=\frac{\left(b^{2}+w^{2}\right)^{1 / 2}}{w}, \quad c_{2}=\frac{-1}{w}, \quad c_{3}=\frac{a_{0}}{w}, \quad c_{4}=\frac{\left[\left(a_{1}-b\right)^{2}+w^{2}\right]^{1 / 2}}{w} \\
w=\left(a_{0}-b^{2}\right)^{1 / 2}, \quad b=\frac{a_{1}}{2}, \quad \psi_{1}=\tan ^{-1}\left(\frac{w}{-b}\right),
\end{gathered}
$$

and

$$
\psi_{2}=\tan ^{-1}\left(\frac{w}{a_{1}-b}\right) .
$$

In this example we take the columns of the state transition matrix $\Phi\left(t, t_{0}\right)$ as $\phi_{1}(t)$ and $\phi_{2}(t)$ and the vectors $f_{1}(t)$ and $f_{2}(t)$ as the rows of the matrix $\Phi\left(t_{0}, t\right) B$. We consider the control function $u(t)$ as the element in the Hilbert function space $H_{1}=L_{2}(\tau)$. It is not difficult to show that $(2,8)$

$$
\begin{aligned}
\beta & =\Phi\left(t_{0}, T\right)\left[x(T)-\Phi\left(T, t_{0}\right) x\left(t_{0}\right)\right] \\
\mathcal{F} & =\int_{t_{0}}^{T} \Phi\left(t_{0}, s\right) B B^{*} \Phi^{*}\left(t_{0}, s\right) d s \\
& =\left[\begin{array}{ll}
f_{11} & f_{12} \\
f_{21} & f_{22}
\end{array}\right]
\end{aligned}
$$

where

$$
\begin{aligned}
& f_{11}=\int_{t_{0}}^{T} d^{2} \phi_{12}{ }^{2}\left(t_{0}, s\right) d s \\
& f_{12}=\int_{t_{0}}^{T} d^{2} \phi_{12}\left(t_{0}, s\right) \phi_{22}\left(t_{0}, s\right) d s \\
& f_{21}=\int_{t_{0}}^{T^{T}} d^{2} \phi_{22}\left(t_{0}, s\right) \phi_{12}\left(t_{0}, s\right) d s \\
& f_{22}=\int_{t_{0}}^{T} d^{2} \phi_{22}{ }^{2}\left(t_{0}, s\right) d s .
\end{aligned}
$$


The optimal control function $u^{0}(t)$ or $\theta_{i}{ }^{0}(t)$ is given by

$$
\begin{aligned}
u^{0}(t) & =B^{*} \Phi^{*}\left(t_{0}, t\right) \mathcal{F}^{-1} \beta \\
& =\frac{d}{\left(f_{11} f_{22}-f_{12} f_{21}\right)}\left(d_{1} f_{22} \phi_{12}-d_{2} f_{12} \phi_{12}-d_{1} f_{21} \phi_{22}+d_{2} f_{11} \phi_{22}\right)
\end{aligned}
$$

and the optimal performance index is

$$
\begin{aligned}
J^{0} & =\left\langle\beta, \mathfrak{F}^{-1} \beta\right\rangle \\
& =\frac{1}{\left(f_{11} f_{22}-f_{12} f_{21}\right)}\left(d_{1}^{2} f_{22}-d_{1} d_{2} f_{12}-d_{1} d_{2} f_{21}+d_{2}^{2} f_{11}\right) .
\end{aligned}
$$

Given specific values of these constants $J, f, R, A, k_{1}, k_{2}, k_{3}$ and $k_{4}$ we can compute $\theta^{0}(t)$ and $J^{0}$. This method is applicable to high order systems, only the size of the related transition matrix increases.

\section{Example II.}

Given a plant whose dynamic characteristic is defined by

$$
\dot{x}(t)=A(t) x(t)+B(t) u(t), \quad t \in \tau=\left[t_{0}, T\right]
$$

and the reference trajectory $\hat{x}(t)$. Find the control function $u$ such that $x(t)$ transfers from $x\left(t_{0}\right)=0$ to $x(T)=x_{T}$, and

$$
J(u)=\int_{t_{0}}^{T}\{[(x-\hat{x}),(x-\hat{x})]+[u, u]\} d s
$$

is minimized.

We may reformulate the above problem as follows: Given $\hat{x} \in H_{1}$ and $x(t) \in R^{n}$ and let $F^{t}$, defined by Eq. 50, be the transformation from $H_{1}=$ $\left[L_{2}(\tau)\right]^{m}$ onto $R^{n}$ to find the element $u \in H_{1}$ such that

is minimized.

$$
J(u)=\|x-\hat{x}\|_{H^{2}}^{2}+\|u\|_{H 1}^{2}
$$

For the present case $\hat{u}=0$ and $(\hat{x}, 0)$ is a tuplet of $H_{1} \times H_{2}$. The relation $F x=u$ does not hold. Theorem $\mathrm{V}$, therefore, is applied. The element $u^{0}$ is given by Eq. 38 or, after transformation, Eq. 69.

\section{Conclusion}

Minimum energy problems in Hilbert function space for three types of index of performance have been formulated. A method of evaluating the optimal element $u^{0}$ without the inverse of the operator $\left(I+F^{*} F\right)$ has been studied.

These methods are applicable to both continuous and discrete linear systems. In the continuous case we consider $H=\left[L_{2}(\tau)\right]^{m}, t \in \tau=\left[t_{0}, T\right]$ and in the discrete case, $H=\left[l_{2}(\sigma)\right]^{m}, \sigma=\left[t_{0}, t_{1}, \cdots t_{f}\right]$. Two examples of continuous 
linear systems have been chosen for the purpose of illustration. Discrete linear systems can be similarly described.

\section{Appendix $A$}

A Proof of Lemmas 2, 3, and 4. Lemma 2 follows directly from the definition. To prove Lemma 3 we note that the function

$$
\left\langle\left(f_{1}, g_{1}\right),\left(f_{2}, g_{2}\right)\right\rangle_{H(F)}=\left\langle f_{1}, f_{2}\right\rangle_{H_{1}}+\left\langle g_{1}, g_{2}\right\rangle_{H_{2}}
$$

is an inner product on $H(F)$ as is easily shown. For instance, if $w_{1}=\left(f_{1}, g_{1}\right)$ and $w_{2}=\left(f_{2}, g_{2}\right)$ then

$$
\begin{aligned}
\left(w_{1}, w_{2}\right\rangle_{H(F)} & \left.=\left\langle f_{1}, f_{2}\right\rangle_{H_{1}}+\left\langle g_{1}, g_{2}\right\rangle_{H_{2}}=\left\langle\overline{f_{2}, f_{1}}\right\rangle_{H_{1}}+\overline{\left\langle g_{2}, g_{1}\right.}\right\rangle_{H_{2}} \\
& =\left\langle\overline{w_{2}, w_{1}}\right\rangle_{H(F)}
\end{aligned}
$$

where $\bar{w}$ denotes the complex conjugate of $w$. Thus, it remains only to show that $H(F)$ is complete in order to prove that $H(F)$ is a Hilbert space. Let $\left\{w_{n}=\right.$ $\left.\left(f_{n}, g_{n}\right)\right\}$ denote a Cauchy sequence in $H(F)$ then for arbitrary $e$ there exists $N$ such that

$$
\left\|w_{m}-w_{n}\right\|_{H^{(F)}}^{2}=\left\|f_{m}-f_{n}\right\|_{H_{1}}^{2}+\left\|g_{m}-g_{n}\right\|_{H_{2}}^{2}<e \quad \text { for } \quad m, n>N
$$

Clearly, this implies both

$$
\begin{aligned}
\left\|f_{m}-f_{n}\right\|_{H 1}^{2}<e_{1} & \text { and }\left\|g_{m}-g_{n}\right\|_{H 2}^{2}<e_{2} \\
e_{1}+e_{2}=e, & \text { for } m, n>N .
\end{aligned}
$$

Since $H_{1}$ and $H_{2}$ are both complete the sequence $f_{n}$ and $g_{n}$ both converge to points $f$ and $g$ of those spaces. Since $g_{n}=F f_{n}$ for all $n$ it follows that $g=F f$ and hence the element $w$ which is the limit of the sequence $\left\{w_{n}\right\}$ is given by $w=(f, g) \in H(F)$ and henec $H(F)$ is complctc.

To prove Lemma 4 , let $\left(u_{1}, F u_{1}\right),\left(u_{2}, F u_{2}\right) \cdots$ be the sequence of points of $H(F)$. Since $F$ is a bounded linear operator this implies that $F$ is continuous, therefore

$$
F u_{n} \rightarrow F u \quad \text { as } \quad u_{n} \rightarrow u \text {. }
$$

This leads to

$$
\lim _{n \rightarrow \infty}\left(u_{n}, F u_{n}\right)=(u, F u) \in H(F) \subset H_{1} \times H_{2} .
$$

We must show that $H(F)$ is closed or equivalently that its complement, denoted by $H(F)^{c}$, is open. If $H(F)^{c}$ is not open then there must be a point $(c, F c) \in H(F)^{c}$ such that for each neighborhood $N$ of $(c, F c)$

$$
N \subset H(F)^{c} .
$$


In particular, the sphere $S((c, F c), 1 / n) \propto H(F)^{\mathrm{c}}$ for $n=1,2,3, \cdots$. Thus, there is a sequence of points $\left(u_{1}, F u_{1}\right),\left(u_{2}, F u_{2}\right) \cdots$ such that

$$
\left(u_{n}, F u_{n}\right) \in H(F) \text { and } \lim _{n \rightarrow \infty}\left(u_{n}, F u_{n}\right)=(c, F c) \in H(F)
$$

which contradicts to the assumption that $(c, F c) \in H(F)^{c}$. Therefore, $H(F)^{c}$ is open and $H(F)$ is closed.

\section{Appendix $B$}

A Proof of $H(F) \perp=V H\left(F^{*}\right)$. Let $(x, y)$ be an arbitrary element of $H(F) \perp$, then for every $u \in H_{1}$

$$
\begin{aligned}
\mathbf{0} & =\langle(x, y),(u, F u)\rangle_{H(F)}=\langle x, u\rangle_{H_{1}}+\langle y, F u\rangle_{H_{2}} \\
& =\langle x, u\rangle_{H_{1}}+\left\langle F^{*} y, u\right\rangle_{H_{1}}=\left\langle x+F^{*} y, u\right\rangle_{H_{1}}
\end{aligned}
$$

which implies $x=-F^{*} y$ or that $(x, y)$ is of the form

$$
(x, y)=\left(-F^{*} y, y\right)=V\left(y, F^{*} y\right)
$$

Thus, $(x, y) \in V H\left(F^{*}\right)$ and hence $H(F) \perp \subset V H\left(F^{*}\right)$. Conversely, if $(x, y) \in$ $V H\left(F^{*}\right)$ then

$$
(x, y)=V\left(y, F^{*} y\right)=\left(-F^{*} y, y\right)
$$

For any $(u, F u)$, however, we have $(u, F u) \perp\left(-F^{*} y, y\right)$ since

$$
\begin{aligned}
\left\langle(u, F u),\left(-F^{*} y, y\right)\right\rangle_{H(F)} & =\left\langle u,-F^{*} y\right\rangle_{H_{1}}+\langle F u, y\rangle_{H_{2}} \\
& =-\langle F u, y\rangle_{H_{2}}+\langle F u, y\rangle_{H_{2}}=0 .
\end{aligned}
$$

Hence, $\left(-F^{*} y, y\right) \in H(F) \perp$ which implies $V H\left(F^{*}\right) \subset H(F) \perp$, and the lemma is proved.

\section{Appendix $C$}

A proof of Eq. 54. From the definition of adjoint operator we have

$$
\langle x, F y\rangle_{H_{1}}=\left\langle F^{*} x, y\right\rangle_{H_{2}}
$$

In particular, if

$$
H_{1}=H_{2}=L_{2}(\tau) \quad \text { then } \quad\langle x, F y\rangle_{L_{2}}=\left\langle F^{*} x, y\right\rangle_{L_{2}} .
$$

In real space $L_{2}\left(t_{0}, T\right)$ the inner product of two elements $u$ and $v$ is defined by

$$
\langle u, v\rangle_{L_{2}}=\int_{t_{0}}^{T}[u, v] d s .
$$


Thus

$$
\begin{aligned}
\langle x, F y\rangle_{L_{2}}= & \int_{t 0}^{T}\left[x\left(s_{1}\right), \Phi\left(s_{1}, t_{0}\right) \int_{t 0}^{s_{1}} \Phi\left(t_{0}, s_{2}\right) B\left(s_{2}\right) y\left(s_{2}\right) d s_{2}\right] d s_{1} \\
= & \int_{t 0}^{T} \int_{t 0}^{s_{1}} y^{*}\left(s_{2}\right) B^{*}\left(s_{2}\right) \Phi^{*}\left(t_{0}, s_{2}\right) \Phi^{*}\left(s_{1}, t_{0}\right) x\left(s_{1}\right) d s_{2} d s_{1} \\
= & \int_{t 0}^{T} \int_{s_{2}}^{T} y^{*}\left(s_{2}\right) B^{*}\left(s_{2}\right) \Phi^{*}\left(t_{0}, s_{2}\right) \Phi^{*}\left(s_{1}, t_{0}\right) x\left(s_{1}\right) d s_{1} d s_{2} \\
& \left\langle\int_{s_{2}}^{T} B^{*}\left(s_{2}\right) \Phi^{*}\left(t_{0}, s_{2}\right) \Phi^{*}\left(s_{1}, t_{0}\right) x\left(s_{1}\right) d s_{1}, y\left(s_{2}\right)\right\rangle_{L_{2}}
\end{aligned}
$$

From (C-1) and (C-2) we have

$$
\left(F^{*} x\right)\left(s_{2}\right)=\int_{s_{2}}^{T} B^{*}\left(s_{2}\right) \Phi^{*}\left(t_{0}, s_{2}\right) \Phi^{*}\left(s_{1}, t_{0}\right) x\left(s_{1}\right) d s_{1}
$$

or

$$
\left(F^{*} x\right)(t)=B^{*}(t) \Phi^{*}\left(t_{0}, t\right) \int_{t}^{T} \Phi^{*}\left(s, t_{0}\right) x(s) d s .
$$

\section{Acknowledgment}

The authors acknowledge helpful discussions with Professor W. A. Porter of the University of Michigan.

\section{References}

(1) A. V. Balakrishnan, "An Operator Theoretic Formulation of a Class of Control Problems and a Steepest Descent Method of Solution," Jour. S.I.A.M. on Control, Vol. 1, No. 2, 1963.

(2) W. A. Porter, "A New Approach to the General Minimum Energy Problem," Joint Automatic Control Conference, Stanford Univ., Stanford, Calif., 1964.

(3) K. Hoffman and R. Kunze, "Linear Algebra," Englewood Cliffs, N. J., Prentice-Hall, 1961.

(4) G. F. Simmon, "Topology and Modern Analysis," New York, McGraw-Hill Book Co., 1963.

(5) A. N. Kolmogorov and S. V. Fomin, "Functional Analysis," Rochester, N. Y., Graylock Press, 1957.

(6) N. I. Akhiezer and I. M. Glazman, "Theory of Linear Operators in Hilbert Space," New York, Frederick Ungar Pub. Co., 1961.

(7) R. E. Kalman, "Contribution to the Theory of Optimal Control," Bol. Soc. Mat., Mexicana, 1961.

(8) Marshall C. Y. Kuo, "The Application of Functional Analysis to Solve a Class of Linear Control Problems," Ph.D. thesis, Univ. of Mich., Ann Arbor, Mich., Dec. 1964. 